\title{
Gestión de aula como estrategia orientadora en el proceso enseñanza aprendizaje
}

\section{Classroom management as a guiding strategy in the teaching of the human condition}

\author{
Edilberto Hart Montes \\ edilberto1217@hotmail.com \\ Universidad del Atlántico \\ Colombia \\ https://orcid.org/0000-0002-3474-8709 \\ Carlos Mario Ramos Gelvez \\ carlosramosgelvez@hotmail.com \\ Universidad del Atlántico \\ Colombia \\ https://orcid.org/0000-0003-2718-3591
}

Recibido: 29 de septiembre de 2019

Aprobado: 1 de noviembre de 2019

\section{RESUMEN}

El propósito fundamental de esta investigación fue comprender la gestión de aula como estrategia orientadora en la enseñanza de la condición humana. Basada desde la naturaleza cualitativa, manejando perspectiva de análisis documental. La educación se constituye en una fuente de pensamiento que hace de la reflexión, una cultura, donde la educación y las prácticas pedagógicas se entretejen por unos enunciados básicos, dando sentido a la identidad; la educación tiene fundamentación ética y política, prácticas sociales arraigadas que giran en torno a la posibilidad de ser aprendidas y desarrolladas para pensar y resolver problemas educativos y sociales. Se considera de vital importancia que los maestros en formación y en ejercicio inicien y profundicen en procesos de reflexión permanente sobre su quehacer en el aula para generar praxis pedagógica.

Descriptores: Gestión de aula; estrategia orientadora; proceso enseñanza aprendizaje; naturaleza cualitativa.

\section{ABSTRACT}

The fundamental purpose of this research was to understand classroom management as a guiding strategy in the teaching of the human condition. Based on 
the qualitative nature, managing the perspective of documentary analysis. Education is a source of thought that makes reflection, a culture, where education and pedagogical practices are interwoven by some basic statements, giving meaning to identity; Education has an ethical and political foundation, entrenched social practices that revolve around the possibility of being learned and developed to think and solve educational and social problems. It is considered of vital importance that teachers in training and in practice initiate and deepen in processes of permanent reflection on their work in the classroom to generate pedagogical praxis.

Descriptors: Classroom management; guiding strategy; teaching learning process; qualitative nature.

\section{INTRODUCCIÓN}

Los cambios que suceden a nivel mundial y sobre todo en América Latina, producto de la globalización, la sociedad de la información y la ola tecnológica, hacen propicio preguntarse sobre el quehacer de los profesores para mejorar la calidad de la educación, si realmente se tiene en cuenta que en el mundo todo está relacionado, nada está aislado; la escuela necesita viabilizar cambios pertinentes en los integrantes de la comunidad educativa y en el entorno, de tal manera que respondan en forma positiva a las exigencias del siglo XXI. Lo anterior lleva a pensar que en la actualidad se está bastante lejos de haber resuelto el problema de la educación, no sólo en cuestión de la tecnología, sino en enfatizar sobre los medios para obtener mejores resultados.

¿Para qué se enseña?, ¿cómo se enseña? $Y$ en función de ello ¿qué se ha de enseñar?, y por supuesto ¿cuál es la mejor manera de enseñar? Todos estos interrogantes conducen a reflexionar sobre las relaciones que se está llevando a cabo entre el sujeto que enseña -profesor- y el sujeto que aprende -estudiante-, es decir, una reflexión sobre las prácticas pedagógicas.

El maestro que reúna las condiciones exigidas, es aquel que se ofrece como puente que tiende a sus estudiantes a fin de que, a invitación suya, puedan cruzarlo. Sin embargo, una vez que con su ayuda lo han franqueado, el puente se viene abajo clamorosamente. El maestro les alienta entonces, a construir sus propios puentes (Correa, 2009). Es así como el profesor, desde su práctica pedagógica, está llamado a reflexionar sobre: su proceso de planeación, la relación y el estilo pedagógico, y el 


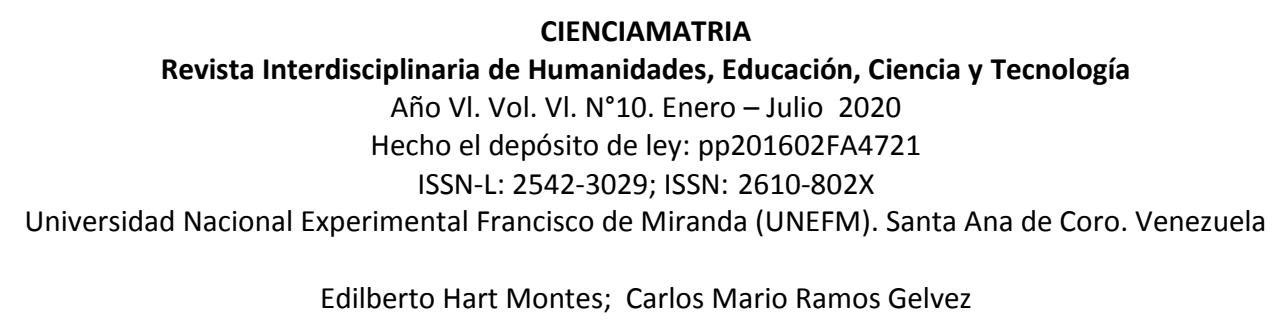

proceso de evaluación que lleva a cabo dentro de su quehacer.

Por lo tanto, la educación se constituye en una fuente de pensamiento que hace de la reflexión una cultura, donde la educación y las prácticas pedagógicas se entretejen por unos enunciados básicos, que le dan sentido a la identidad, tienen una fundamentación ética y política, arraigadas prácticas sociales que giran en torno a la posibilidad de ser aprendida y desarrolladas para pensar y resolver problemas educativos y sociales.

Se considera de vital importancia que los maestros en formación y en ejercicio inicien y profundicen en procesos de reflexión permanente sobre su quehacer en el aula para generar praxis pedagógica, como acto cotidiano de construcción y reconstrucción del quehacer educativo, que permita ampliar las experiencias del momento, comprender los contextos y establecer un análisis crítico para estructurar, relaborar y organizar nuevos saberes, que a su vez, los convalida, consolida y transforma en su práctica pedagógica.

\section{DESARROLLO TEÓRICO}

\section{El profesor un profesional de la educación}

Aplicar el currículo y hacerlo efectivo en la práctica, debe ser una prioridad a la hora de gestionar el trabajo en aula. La gestión de aula se centra en generar oportunidades efectivas en la sala de clases, programando unidades didácticas coherentes, tomando decisiones asertivas, contextualizadas y secuenciadas, organizando y planificando acorde con el sujeto que aprende, los contenidos, la opción metodológica, la evaluación, el contexto y los recursos didácticos; sin olvidar, por cierto, las presiones y problemas que lo afectan. No es fácil, sobre todo en la complejidad de la tarea de enseñar que se encuentra afectada por innumerables desafíos dentro del sistema.

Ser un profesional de la educación significa contar con conocimientos especializados, tener compromiso frente a la tarea que se ejerce controlando la práctica en autonomía profesional, en el fondo, como señala Le Boterf (2009) un profesional sabe gestionar una situación profesional compleja, sabiendo actuar y reaccionar con pertinencia, combinar los recursos y movilizarlos en un contexto, 


CIENCIAMATRIA
Revista Interdisciplinaria de Humanidades, Educación, Ciencia y Tecnología
Año VI. Vol. VI. N${ }^{\circ}$. Enero - Julio 2020
Hecho el depósito de ley: pp201602FA4721
ISSN-L: 2542-3029; ISSN: 2610-802X
Universidad Nacional Experimental Francisco de Miranda (UNEFM). Santa Ana de Coro. Venezuela
Edilberto Hart Montes; Carlos Mario Ramos Gelvez

sabe transferir y utilizar sus meta conocimientos para modelar e interpretar los indicadores en contexto, sabe aprender y aprender a aprender y sabe comprometerse.

Es así como, gestionar situaciones complejas ya es casi un lema profesional, pues, hoy por hoy, estamos insertos en una sociedad que requiere del aprendizaje y de la enseñanza para la generación de nuevos conocimientos, pero en realidad es muy poco lo que se ha logrado avanzar en este tema. Las interacciones que se desarrollan hoy en día entre estudiantes y profesores y la forma en que se está enseñando han tenido muy pocas modificaciones y cambios, pues aún se siguen repitiendo patrones y conductas, y por el contrario se han agregado nuevas dificultades o desafíos como la desvaloración de los sistemas (familia, escuela, religión, sociedad) con el consiguiente desinterés por el estudio y los valores del esfuerzo por parte de los estudiantes.

A su vez, la generación de conocimientos parcelados, fragmentados por efecto de la saturación de información, sonidos e imágenes que no se cuestionan dentro de un pensamiento reflexivo y autónomo. Todo esto no es un tema menor y nos da a entender que estamos dentro de una sociedad con nuevos escenarios, nuevos lenguajes y nuevos actores que queramos o no, afectan en la sala de clases.

\section{Gestión de Aula}

El término gestión de aula ha sido ampliamente discutido. Actualmente ha pasado de comprenderse desde una mirada técnica, centrada en estrategias para mantener el silencio y el control de los estudiantes, a una gestión altamente compleja para diseñar un ambiente de aprendizaje basado en la sana convivencia y las relaciones interpersonales.

En este sentido, se sabe que el rol del profesor es clave para la efectividad de la enseñanza, es decir, para lograr que todos los estudiantes desarrollen las competencias y adquieran los conocimientos esperados. Las investigaciones en esta materia han evidenciado que la enseñanza y el aprendizaje no pueden ocurrir en una clase con manejo deficiente del aula (Hattie, 2009). 


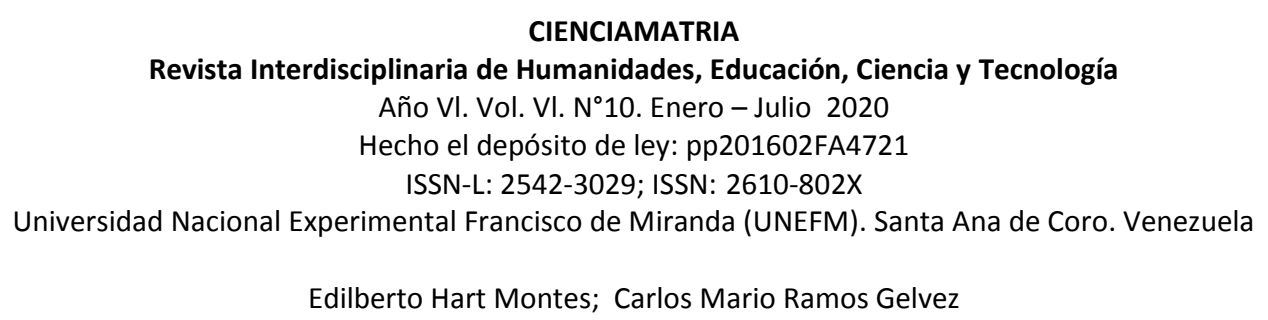

Pero ¿Qué es la gestión de aula? La gestión de aula envuelve un conjunto de acciones de variada índole que permiten construir un contexto propicio para el aprendizaje. Estas acciones van desde la organización de los contenidos y el diseño instruccional propiamente tal, hasta la promoción de interacciones pacíficas y colaborativas entre los estudiantes.

Asimismo, Brophy (2006) plantea que la gestión de aula implica todas aquellas acciones que permiten crear y mantener un ambiente de aprendizaje donde se articulan instrucciones que potencian la construcción y organización de un ambiente físico del aula, estableciendo reglas y procedimientos que posibilitan la atención de los estudiantes.

\section{Concepciones de enseñanza}

Cuanto más profundo son los cambios que experimenta la sociedad, mayor es la importancia de la educación mayor es la responsabilidad para con los ciudadanos, desde las diversas áreas de gestión de las instituciones educativas, se trabajan para el desarrollo de las diversas dimensiones con el propósito central que estos ciudadanos tengan mayor compromiso en continuar empujando el desarrollo de sus naciones.

Asimismo, implica que las organizaciones escolares apoyadas en concepciones de enseñanza entren a acompañar el quehacer docente que le van a permitir a los estudiante adquirir y desarrollar capacidades afectivas, sociales, emocionales, cognitivas y cognoscitivas para hacerte frentes a los obstáculos que la misma cultura y sociedad va imponiendo en la medida el niño y joven se desarrollarse como sujeto de derecho, que desea como persona disfrutar de las mieles del éxito a la cual todos tienen derecho.

En ese sentido, es importante hacer mención a Urueta (2019) cuando señala que las estrategias son conscientes e intencionales, dirigidas a un objetivo relacionado con los procesos formativos. Son consideradas procesos mediante los cuales se eligen, coordinan, aplican habilidades, que se vinculan con la enseñanza y el aprendizaje. Dependen de los objetivos y contenidos que se persigan. 


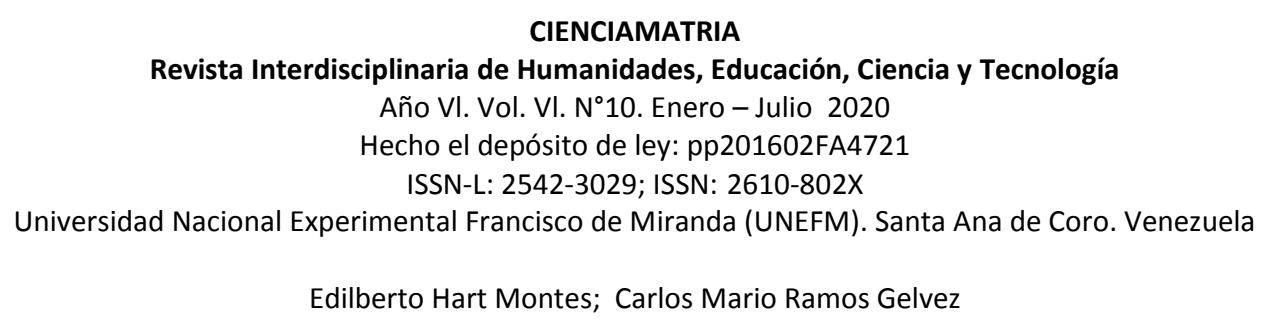

Por su parte la labor del maestro, necesita trascender la trasmisión de conocimientos e información a los estudiantes, de acuerdo a la mirada de Ramsden (2013) uno de sus objetivos es lograr la comprensión de la materia, lo cual es posible si se facilita el trabajo cooperativo como estrategia de aprendizaje. Desde esta concepción, el maestro le implica asumir una postura humanista como es la de conocer al niño y al joven como criatura humana, las dificultades e incomprensiones de estos para acercarse a los saberes con la finalidad de intervenir y así cambiarlas.

Para lograr las nuevas alteraciones en las estructuras mentales y humanos en el sujeto educable, es de suma obligatoriedad crear entornos de aprendizaje que anime activamente a los estudiantes a implicarse en la maduración de sus inteligencias y al empoderamiento de nuevos saberes necesarios para ser más audaz en la comprensión del conocimiento de sí mismo y darle mayor connotación a su vida y la vida de los otros.

Desde la postura conceptual Freire (2014) el educador es un agente transformador, lo que implica desde el aula escolar asumir una coherencia contundente entre el discurso conceptual con la praxis desarrollada en el aula de clase, que su accionar este siempre apuntando a la realización del proyecto de hombre, que le apueste a no perder a pesar de su humildad como humanos, que no pierda la capacidad de asombro, de maravillarse por lo que vive, siente y hace, y sobre todo tenga la capacidad de anhelo es decir de ser él y no lo que otros desean de él, ser un maestro que en el aula siembre en los estudiantes la pasión por la autonomía, la libertad, que se le rinda tributo al libre albedrío.

Hace saber Valero y Cortes (2013) que el desarrollo de actividades educativas significativas, llevan al estudiante a relacionar e integrar la nueva información con sus estructuras mentales, posibilitando la existencia de un aprendizaje significativo. Saberes que deben trascender; estos se constituyen en nuevos insumos para el desarrollo de su inteligencia.

Cuando el maestro logra la comunión entre los nuevos saberes que fueron acomodados exitosamente en sus estructuras, con los intereses de los estudiantes y los propósitos que implica educar para el mundo de hoy, se derrumban las barraras que impiden que los estudiantes desplieguen los dones con que vino equipada su 


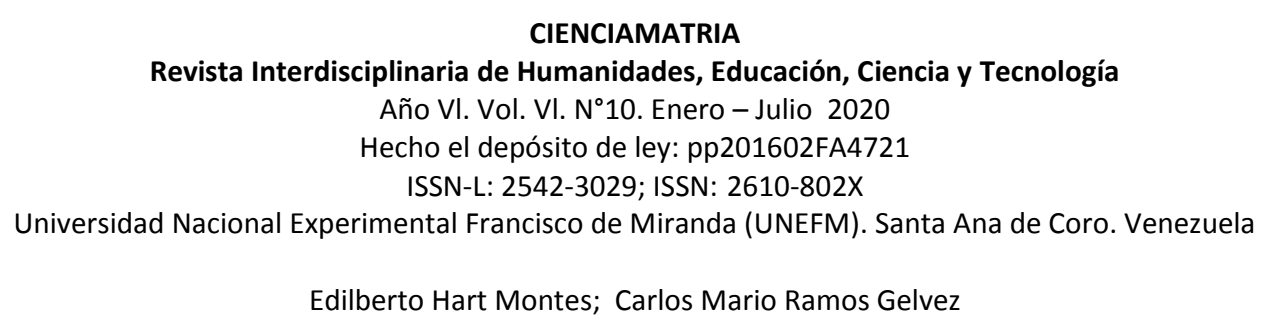

naturaleza humana, de lo contrario, el maestro se convertirá en un torpe e irreflexible de su labor de labrar en los estudiantes la inteligencia.

De acuerdo con Freire (2014) el educador es un profesional que trabaja por la superación de las injusticias sociales, que trata a los estudiantes como sujetos críticos, que se preocupa por hacer problemático el conocimiento, que recurre al diálogo crítico y afirmativo y, en definitiva, que lucha por un mundo cualitativamente mejor para todas las personas.

Todo ello supone para el profesor ayudar al estudiante a ser un sujeto integrado, creador, ayudarlo examinar, entender los valores, las influencias que le rodean, estar alerta a sus propios pensamientos y sentimientos, consciente que está condicionado, consciente de sus acciones y reacciones, es decir su actitud que se convierte en una señal innegable que su inteligencia está en pleno desarrollo, lo que también desemboca en una mejor relación con las personas y con el mundo de las cosas.

En este sentido, Mendoza (2006), es de la concepción de que el maestro está consciente de sus limitantes, reconoce que no lo sabe todo y que incluso lo que sabe puede llegar a comprenderlo mejor. De este modo cultivar el saber se convierte en una tarea de toda la vida. Al maestro de hoy, se debe actualizar constante mente, no hay otra alternativa, de lo contrario vera el mundo de la misma manera y no como es.

Esa renovación permanente lo llevara entender que la inteligencia no solo se limita almacenar información o contestar un examen, sino aquella que se hace al hombre fuerte, libre, disfrutar de la vida, disfrutar del otro. Si se quiere que los estudiantes tengan una educación de alto nivel, es el maestro el primero que debe superar la infelicidad, los temores, las frustraciones de los cuales ningún hombre se escapa.

El maestro es sabedor que todo su vida profesional estará inmerso en un proceso de continua formación, es decir el desaprender y el reaprender es de mayor relevancia en la actualidad que el simple aprender en razón que en la ciencia no existen dogmas y quienes asumen este rol de maestro dogmático se convierten en un obstáculos para desarrollar procesos educativos que fortalezcan las relaciones consigo mismo, con el otro y con el medio, lo es considerado en el actual mundo como algo crucial para construir puentes de convivencia y de paz entre las personas 


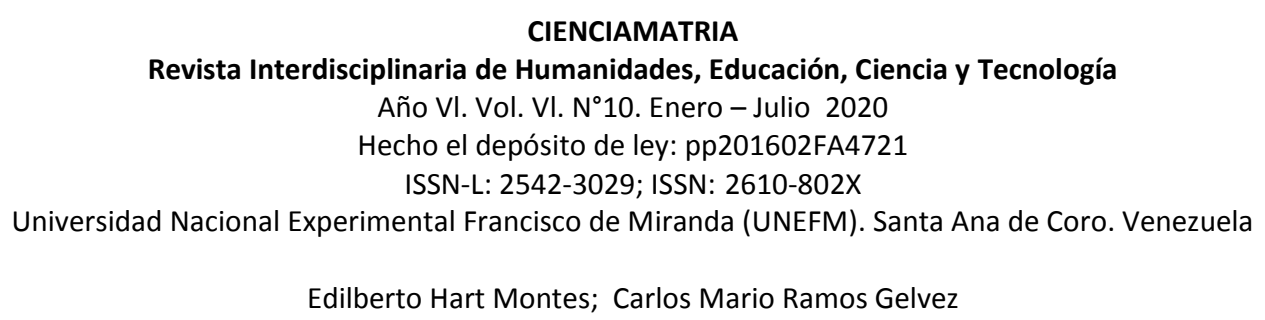

y naciones.

El maestro que vive su vocación plenamente, sabe sacar provecho de cada acto desarrollado con los estudiantes, el aula, se convierte en un escenario de aprendizaje aprendiendo de forma constante de ellas, indiscutiblemente según a juicio del autor citado tiene mucho que dar, su personalidad misma resulta atractiva para los estudiantes y su vida se convierte en un libro abierto que nunca pasa de moda y del que es posible aprender mucho cada instante.

Mendoza (2006) considera que el maestro que vive su vocación, que enseña con pasión cumple con el propósito que se ha marcado en su vida profesional. El maestro que arrastra con su ejemplo ayuda a los estudiantes a encontrar el sentido que tiene la vida. Este tipo de maestro le facilitara al estudiante sacar mejor provecho de las oportunidades educativas que le ofrece su maestro. Quien tiene la vocación autentica, disfrutan de sus responsabilidades, se muestran tranquilo frente a las adversidades y cualquier imprevisto se convierte en un reto, en una situación de prueba para la mejora continua.

\section{Estrategias para el Aprendizaje}

El proceso de enseñanza y aprendizaje es una construcción conjunta que responde a un continuo complejo intercambio entre el docente, los alumnos y el contexto tanto en el aula con su ambiente de aprendizaje, como en la realidad en la cual se desarrolla la actividad, Díaz, Barriga y Hernández, (2009, p.7), definen las estrategias docentes como "las actividades que realiza el docente en el manejo de una serie de métodos y técnicas, rígidas o flexibles y adaptables a las diferencias de sus alumnos, así como al contexto de su clase", por ello existen diversas vías para promover el aprendizaje, el docente ha de ser capaz de decidir en cada caso.

Las estrategias docentes son procedimientos utilizados por el profesor en forma rígida o flexible para promover la reflexión en el logro del aprendizaje en sus alumnos, empleando para tal fin todos los medios y recursos necesarios, esto según el enfoque en el cual se sustenta su desempeño académico, sea este conductista, constructivista o ecléctico, para ello el docente debe tener conciencia de la teoría en la que apoya su trabajo en el aula. 


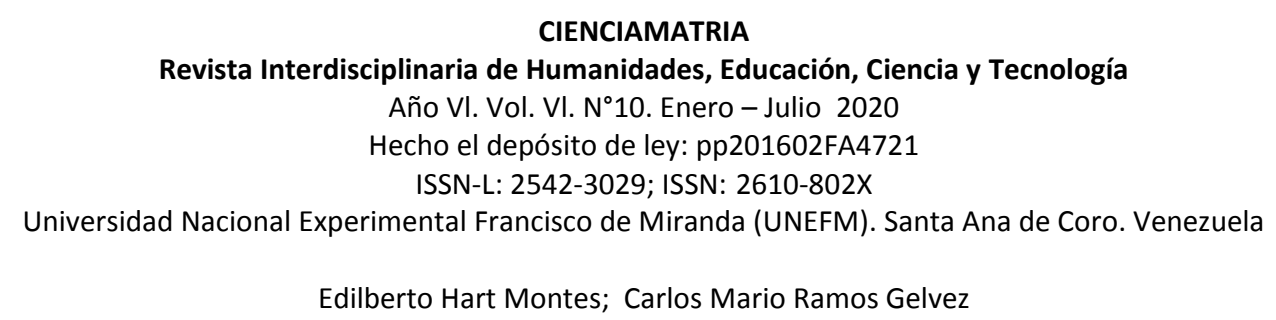

Para la elaboración e implementación de las estrategias docentes, Díaz, Barriga y Hernández (2009, p.26), consideran que es necesario tener en cuenta: "las características generales de los alumnos (nivel de desarrollo cognitivo, factores afectivos, entre otros), el conocimiento previo y el curricular, la meta que se desea lograr, las actividades de aprendizaje, la evaluación del progreso del aprendizaje del estudiante", se debe agregar la determinación del contexto intersubjetivo, creado en conjunto con alumnos y el resto de los actores que participan en el proceso de enseñanza y de aprendizaje, este aspecto depende del enfoque psicoeducativo adoptado.

\section{METODOLOGÍA}

Se implementó un estudio cualitativo basado en el análisis documental que según Pinto y Gálvez, (2016) consiste en buscar, seleccionar, organizar y analizar un conjunto de materiales escritos para responder una o varias preguntas sobre un tema. En el presente estudio se analizaron una serie de documentos en torno al tema gestión de aula como estrategia orientadora en la enseñanza de la condición humana.

Asimismo, Alfonso (2015) define la investigación documental como el proceso de construcción de conocimientos a partir de un trabajo sistemático de indagación, recolección, organización, análisis e interpretación de información en torno a un tema seleccionado y delimitado. En este caso, se parte de la lectura, análisis, reflexión e interpretación de artículos científicos pertinentes y significativos publicados en revistas indexadas encontradas en diferentes bases de datos sobre la gestión de aula como estrategia orientadora en la enseñanza de la condición humana.

\section{REFLEXIONES FINALES}

Se concluye que si una de las funciones de la educación es promover la capacidad de los alumnos para gestionar sus propios aprendizajes, adoptar una autonomía creciente en su carrera académica y disponer de herramientas intelectuales y sociales que les permitan un aprendizaje continuo a lo largo de toda su vida, los 


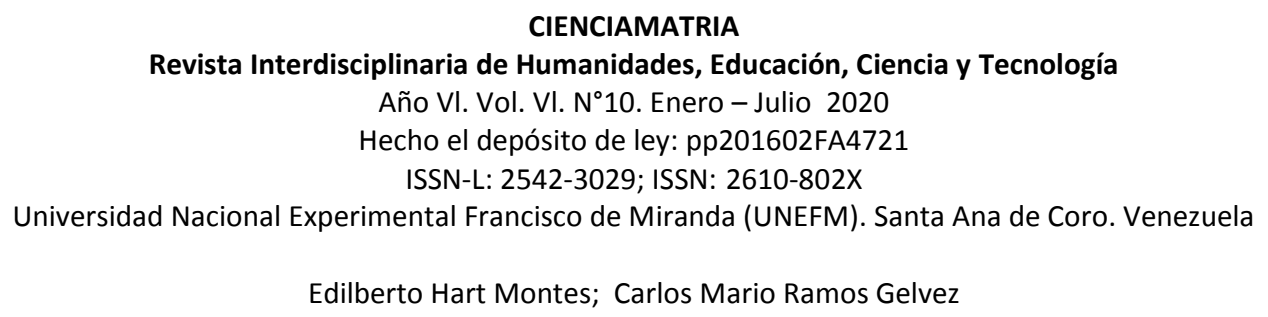

profesores tenemos que crear condiciones en el contexto del aula universitaria que ayuden a los alumnos a adquirir estrategias y capacidades que les permitan transformar, reelaborar y, en suma, reconstruir los conocimientos que reciben.

En el mismo orden de ideas, el proceso enseñanza-aprendizaje constituye un verdadero par dialéctico en el cual el primer componente debe organizarse y desarrollarse de manera tal que facilite la apropiación del conocimiento de la realidad objetiva que, en su interacción con un sustrato material neuronal, asentado en el subsistema nervioso central del individuo, permitirá que en el menor tiempo y con el mayor grado de eficiencia y eficacia posibles, el establecimiento de los engramas sensoriales, aspectos intelectivos y motores necesarios para que el reflejo se materialice y concrete.

\section{REFERENCIAS CONSULTADAS}

1. Alfonzo, I. (2015). Técnicas de investigación bibliográfica. Caracas: Contexto

2. Brophy, J. (2006). Observational Research on Generic Aspects of Classroom Teaching.

3. Correa, C. (2009). Aprender y Enseñar en el siglo XXI. Bogotá D.C., Colombia: Editorial Magisterio.

4. Díaz Barriga A y Hernández G. (2009). Estrategias docentes para un aprendizaje significativo. Una interpretación constructivista. México: McGrawHill. Ediciones.

5. Freire, P. (2014). La educación como práctica de la libertad. México, D.F: Editorial Siglo Veintiuno.

6. Hattie, J. A. (2009). Visible learning: A synthesis of $800+$ meta-analyses on achievement. Abingdon: Routledge.

7. Le Boterf, G. (2009). Ingeniería de las competencias. Colección formación y desarrollo (p. 461). Barcelona: Gestión 2000.

8. Mendoza, A. (2006). La educación literaria. Bases para la formación de la competencia lecto-literaria. Málaga: Aljibe.

9. Ramsden, P. (2013). Context and Strategies. Situational Influences on Learning. En R. Schmeck (ed.): Learning Strategies and Learning Styles, pp. 159-184. New York: Plenum Press. 


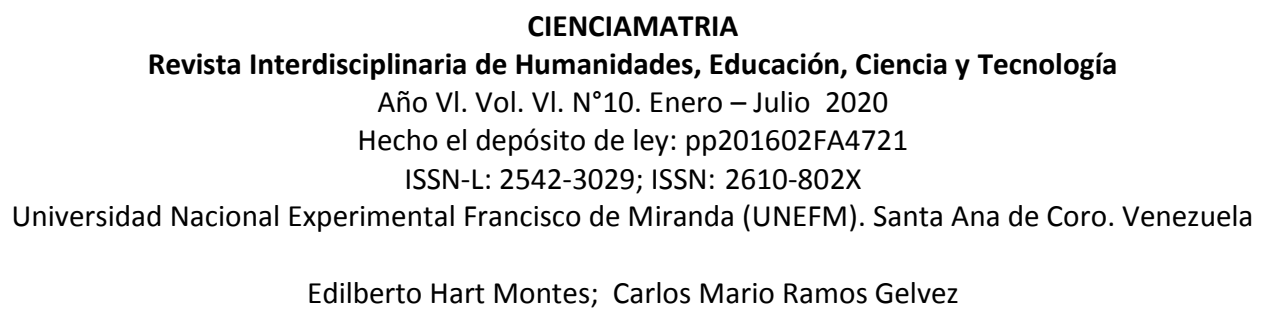

10. Urueta, L (2019) Estrategias de enseñanza y el uso de las tecnologías de información y comunicación en las instituciones educativas departamentales en el Municipio Zona Bananera - Colombia. KOINONIA. Revista Arbitrada Interdisciplinaria de Ciencias de la Educación, Turismo, Ciencias Sociales y Económica, Ciencias del Agro y Mar y Ciencias Exactas y aplicadas. Año IV. Vol IV. N7. Enero - Junio 2019. ISSN: 2542-3088 Fundación Koinonia (F.K). Santa Ana de Coro. Venezuela.

11. Valero, V; Cortés, G (2013) "Inglés I, experiencias con el primer curso en línea de la Coordinación de Lenguas Extranjeras de la UAM-A" [recurso electrónico]. -- p. 417-438. -- En: Coloquio sobre la Práctica de la Educación Virtual en la UAM-A (1‥ : 2012 : UAM Azcapotzalco, Ciudad de México).

\section{REFERENCES CONSULTED}

1. Alfonzo, I. (2015). Literature research techniques. Caracas: Context

2. Brophy, J. (2006). Observational Research on Generic Aspects of Classroom Teaching.

3. Correa, C. (2009). Learn and teach in the 21st century. Bogotá D.C., Colombia: Editorial Magisterio.

4. Díaz Barriga A and Hernández G. (2009). Teaching strategies for meaningful learning. A constructivist interpretation. Mexico: McGraw-Hill. Editions

5. Freire, P. (2014). Education as a practice of freedom. Mexico, D.F: Twentyfirst Century Editorial.

6. Hattie, J. A. (2009). Visible learning: A synthesis of $800+$ meta-analyzes on achievement. Abingdon: Routledge.

7. Le Boterf, G. (2009). Engineering of the competitions. Training and development collection (p. 461). Barcelona: Management 2000.

8. Mendoza, A. (2006). Literary education Bases for the formation of literary competence. Malaga: cistern.

9. Ramsden, P. (2013). Context and Strategies. Situational Influences on Learning. In R. Schmeck (ed.): Learning Strategies and Learning Styles, pp. 159-184. New York: Plenum Press.

10. Urueta, $L$ (2019) Teaching strategies and the use of information and communication technologies in departmental educational institutions in the 
Revista Interdisciplinaria de Humanidades, Educación, Ciencia y Tecnología

Año VI. Vol. VI. N¹0. Enero - Julio 2020

Hecho el depósito de ley: pp201602FA4721

ISSN-L: 2542-3029; ISSN: 2610-802X

Universidad Nacional Experimental Francisco de Miranda (UNEFM). Santa Ana de Coro. Venezuela

Edilberto Hart Montes; Carlos Mario Ramos Gelvez

Zona Bananera Municipality - Colombia. Koinonia Interdisciplinary Arbitrated Journal of Education, Tourism, Social and Economic Sciences, Agro and Sea Sciences and Exact and Applied Sciences. Year IV Vol IV. No. 7 January June 2019. ISSN: 2542-3088 Koinonia Foundation (F.K). Santa Ana de Coro. Venezuela.

11. Valero, V; Cortés, G (2013) "English I, experiences with the first online course of the Coordination of Foreign Languages of the UAM-A" [electronic resource]. - p. 417-438. - In: Colloquium on the Practice of Virtual Education in the UAMA (1st.: 2012: UAM Azcapotzalco, Mexico City).

(C2020 por los autores. Este artículo es de acceso abierto y distribuido según los términos y condiciones de la licencia Creative Commons Atribución-NoComercial-CompartirIgual 4.0 Internacional (CC BY-NC-SA 4.0)

(https://creativecommons.org/licenses/by-nc-sa/4.0/) 